DO

I N S T I T U T O

$\mathrm{DE}$

M E D I C I N A

\title{
SARS-CoV-2 infections with emphasis on pediatric patients: a
} narrative review

\section{T R O P I C A L}

$\mathrm{DE}$

SÃO PAULO

JOURNAL OF THE SÃO PAULO INSTITUTE OF TROPICAL MEDICINE

Rev Inst Med Trop Sao Paulo. 2020;62:e65

http://dx.doi.org/10.1590/s1678-9946202062065

Page 1, where it reads:

Lacyane Silva Pinto

Should be read:

Lacyane Santos Pinto 